\title{
Persistent Müllerian duct syndrome: A case report and review
}

\author{
XIAOYA REN $^{1}$, DI WU ${ }^{1}$ and CHUNXIU GONG ${ }^{1,2}$ \\ ${ }^{1}$ Department of Endocrinology, Genetics, Metabolism and Adolescent Medicine; ${ }^{2}$ Beijing Key Laboratory for \\ Genetics of Birth Defects, Beijing Children's Hospital, Capital Medical University, Beijing 100045, P.R. China
}

Received April 25, 2017; Accepted August 1, 2017

DOI: $10.3892 / \mathrm{etm} .2017 .5281$

\begin{abstract}
Persistent Müllerian duct syndrome (PMDS) is a rare type of male pseudohermaphroditism caused by a deficiency in anti-Müllerian hormone (AMH) or a defect in its type II receptor. The current study reports the clinical data and results of the genetic analysis of a 17-month-old male diagnosed with PMDS. The clinical manifestations of the patient included a left transverse testicular ectopia and bilateral cryptorchidism. Pelvic ultrasonography indicated two testes on the same left inguinal ring and left inguinal hernia and uterine tissue located at the left rear of the bladder. Karyotype analysis detected a 46,XY chromosome pattern and tests determined that the level of $\mathrm{AMH}$ was increased. Gene sequencing of AMHR-II indicated a compound heterozygous nucleotide variation and identified two novel mutations. The c.1184 (E9) to c.1185 (E9) CT deletion mutant gene originated from the father of the patient. This mutation causes a frameshift resulting in a truncated protein. The c.1388G $>$ A (E10) mutant site was derived from the patient's mother and caused a change in p.463, $\mathrm{R}>\mathrm{H}$, resulting in the alteration of the structure of the protein, which subsequently induced a conformational change in AMHR-II. The results of the current study may help to further understanding of the PMDS genetic profile.
\end{abstract}

\section{Introduction}

Persistent Müllerian duct syndrome (PMDS) is a rare form of internal male pseudohermaphroditism characterized by Müllerian duct (MD) derivatives in the genotypes and phenotypes of males with a 46,XY karyotype $(1,2)$. Normally, Sertoli cells begin to produce anti-Müllerian hormone (AMH) during week 7 of gestation, causing MD regression. However, MDs remain in patients with PMDS, due to a deficiency of

Correspondence to: Dr Chunxiu Gong, Department of Endocrinology, Genetics, Metabolism and Adolescent Medicine, Beijing Children's Hospital, Capital Medical University, 56 Nan Lishi Road, Beijing 100045, P.R. China

E-mail: chunxiugong@vip.163.com

Key words: persistent Müllerian duct syndrome, anti-Müllerian hormone, anti-Müllerian hormone type 2 receptor
AMH or a defect in the AMH type II receptor (AMHR-II) (1). PMDS is complex and anatomically variable, which makes diagnosis challenging. Patients with PMDS are categorized into three types, according to the position of the testes and uterus: i) The female type occurs in $60-70 \%$ of patients, in which the bilateral testes and epididymis are connected to the fallopian tubes in the abdomen, the bilateral testes are in analogue positions to the ovaries and their inguinal sacs remain empty; ii) the Uteri Inguinal type occurs in 20-30\% of patients that exhibit a testis in the hernia sac, or a scrotal testis with a contra lateral testis located in the abdomen; and iii) the transverse testicular ectopia makes up the smallest group as it is only classified in $\sim 10 \%$ of patients where the two testes are present in the same hernia sac along with the uterus and uterine tubes $(3,4)$.

The incidence of PMDS is low, with only 150 cases documented globally (5). Only $\sim 13$ cases have been diagnosed in China, all without the use of genetic analysis $(6,7)$. Due to a lack of distinctive clinical features in its early stages, PMDS is typically diagnosed in children when they are tested for other diseases, including cryptorchidism or inguinal hernia, or in adults when they are tested for sterility or oligospermia. The impact on fertility in adults with PMDS diagnosed and treated during childhood remains unknown. However, according to two case reports, delayed diagnosis at adulthood may cause infertility $(8,9)$. The correct diagnosis of PMDS relies on a systematic endocrine assessment and analysis of mutations in the genes coding for AMH (Fig. 1) and AMHR-II (Fig. 2). At present, the treatment of PMDS consists of repairing the external genitalia, which keeps MD structures in the abdomen intact (4). The current study documents the case of a 17-month-old patient diagnosed with PMDS, and presents a review of the relevant literature on the diagnosis and treatment of PMDS.

\section{Case report}

A 17-month-old male was admitted to Beijing Children's Hospital (Beijing, China) in March 2016 presenting with an empty right scrotum and left inguinal mass. The patient was a full term baby with normal labor and had no siblings. The parents were non-consanguineous with no history of infertility and the patient had normal intelligence. The results of the physical examinations were: Height, $79 \mathrm{~cm}$; weight, $10 \mathrm{~kg}$; proportionate anatomy, no malformation of the head or limbs; pubic hair, Tanner stage I (10); penis size, $4.3 \times 1.5 \mathrm{~cm}$; and a 
normal meatus. However, the two sides of the scrotum were small with few folds, the right scrotum was empty and the top of the left scrotum reached the testis and another soft tissue with no tenderness.

A peripheral venous blood sample $(2 \mathrm{ml})$ was taken from the patient, and cultured for $72 \mathrm{~h}$ at $37^{\circ} \mathrm{C}$ with RPMI-1640 Medium (5 ml; Thermo Fisher Scientific, Inc., Waltham, MA, USA). Dividing blood cells were then obtained and $0.1 \mathrm{ml}$ colchicine (Sigma-Aldrich; Merck KGaA, Darmstadt, Germany) was added to stop cell division. Hypotonic solution was then used to induce cytolysis. The cells were fixed on glass slides with methanol and glacial acetic acid for $20 \mathrm{~min}$ at room temperature, and their structures and quantities were observed under a microscope at x100 magnification with an oil immersion lens following giemsa staining for $8 \mathrm{~min}$ at room temperature. A further 4 blood samples $(2 \mathrm{ml})$ were collected from the median cubital vein, and further serum tests were performed following centrifuging blood samples at 2,200 x $\mathrm{g}$ for $7 \mathrm{~min}$ at $37^{\circ} \mathrm{C}$. Samples were analysed via chemiluminescense and radioimmunoassays, using a Cortisol Radioimmunoassay kit (0304103D4101-1), Adrenocorticotropic Hormone Radioimmunoassay kit (0304103D4001; both Roche Applied Science, Penzberg Germany) and Hepatic kit (AU5800; Beckman Coulter, Inc., Brea, CA, USA). The laboratory results were: Karyotype, 46,XY; sex determining region Y protein +; normal liver and kidney function and normal electrolyte, adrenocorticotropic hormone and cortisol levels. The results of the sex hormone tests indicated that the patient was pre-pubescent (Table I). A pelvic ultrasound indicated that two testicular solid hypoechoic masses $1.2 \times 0.5 \times 0.6$ and $1.4 \times 0.5 \times 0.8 \mathrm{~cm}$ large were present at the entrance of the deep inguinal ring and a normal blood flow signal was detected. There were no testicular masses identified during the examination of the right scrotum, inguinal region, inguinal ring or abdomen. A hyperechoic strip measuring $\sim 1.3 \times 0.7 \times 2.2 \mathrm{~cm}$ was present behind the left side of the bladder and a uterine hyperechoic line was identified inside, which suggested the presence of a dysplastic uterus behind the left side of the bladder. The result suggests a dysplastic uterus behind. Genetic testing was performed in Beijing Key Laboratory for Genetics of Birth Defects by Sanger sequencing of AMHR-II, which indicated a compound heterozygous nucleotide variation. This was performed using KAPA HiFi HotStart ReadyMix (KAPA Biosystems, Inc., Wilmington, MA, USA) and the following primers: c.1184(E9) to c.1185(E9), forward 5'-TGTGTCAACAGTTGTAGCAAT ACC-3', and reverse 5'-GACTGTACCACCCATCCTTACC-3'; and c.1388G $>$ A(E10), forward 5'-ACCCCTAGGACTAAC TGATACCC-3' and reverse 5'-TGAACCAAGGAGTGATGG GAGATA-3'.

The c.1184 (E9) to c.1185 (E9) CT deletion mutant gene originated from the father of the patient. This mutation led to a frameshift change resulting in a truncated protein. The c.1388G $>$ A (E10) mutant site was derived from the patient's mother and caused a change in the p.463, $\mathrm{R}>\mathrm{H}$, resulting in the alteration of protein structure, inducing a conformational change in AMHR-II. This change led to a decrease in AMHR-II binding capacity. This patient underwent a left transverse testicular ectopia orchidopexy and during this, a similar type of uterine tissue was identified between the two testes (Fig. 3).

\section{Discussion}

PMDS was first described by Nilson (11) in 1939 as a rare type of male pseudohermaphroditism characterized by the presence of MD derivatives in genotypic and phenotypic males with a 46,XY chromosome pattern $(1,2,5)$. It is caused by a deficiency in AMH or a defect in AMHR-II (12-14). The patterns of PMDS inheritance include $\mathrm{X}$ linkage, autosomal dominant and autosomal recessive inheritance (14). 'Persistent Müllerian duct syndrome' was used as a search term for case reports and reviews with the PubMed database (www.ncbi.nlm.nih. gov/pubmed), covering 45 articles in foreign languages, and 5 articles in Chinese. In total, there have been $\sim 150$ cases of PMDS reported in adults, the majority in the US, Europe and the Middle East (5). In China, 11 pediatric patients were diagnosed with PMDS during laparoscopic treatment at the Children's Hospital of Fudan University (Shanghai, China) (6) without the use of genetic analysis. Two adult patients were misdiagnosed with '34 years of right inguinal hernia' and ' $>20$ years of left inguinal hernia with an empty right hemiscrotum', respectively, at the First Affiliated Hospital of Nanjing Medical University (Nanjing, China) (7). These misdiagnoses may have been caused by the lack of under-virilism in these two adult patients; however, underdeveloped uteruses were identified during surgical examination and then the patients were subsequently diagnosed with PMDS. The incidence of PMDS is low and there is a number of possible reasons for this: i) Little is known about PMDS and therefore cryptorchidism surgery is rarely considered to be indicative of PMDS when the patients exhibit a normal virilism phenotype; and ii) physicians may lack understanding and knowledge of the structure of MDs (15). There have been four cases of PMDS diagnosed in infants $(4,16)$, however, to the best of our knowledge there have been no studies that have conducted genetic analysis prior to diagnosis. The current study described the clinical data and genetic analysis of a 17-month-old patient diagnosed with PMDS.

The internal genital canal consists of MDs and Wolffian ducts prior to sex differentiation during the first 7 weeks of gestation. The sex differentiation of a normal male depends on testosterone, dihydrotestosterone and $\mathrm{AMH}(17) \mathrm{AMH}$ is a member of the transforming growth factor $\beta$ superfamily with a molecular weight of $140 \mathrm{kDa}$ and is the first hormone to be synthesized by Sertoli cells $(5,18)$. Its physiological function is to induce MD regression during male sex differentiation. Due to the existence of the sex-determining region $\mathrm{Y}$ (SRY) gene in males, undifferentiated gonads develop into testes and immature Sertoli cells begin to secrete AMH to induce the regression of the MDs at the end of week 7 of gestation. AMH expression is not dependent on gonadotropins in the embryo stage; it is activated by SRY9 and then splicing factor-1 (SF-1), GATA binding protein 4 and Wilms tumor 1 to increase transcription (19). Following activation of the hypothalamic-pituitary-gonadal axis, AMH upregulates follicle stimulating hormone (FSH) and downregulates androgen (19). Gonadotropin secretion is low in the week following birth and reaches a peak within 6 months (mini puberty). Sertoli cell proliferation is stimulated by FSH and increases the size of the testes and AMH secretion, which causes AMH levels to peak. Luteinizing hormone then stimulates Leydig cells to produce testosterone, stimulating 


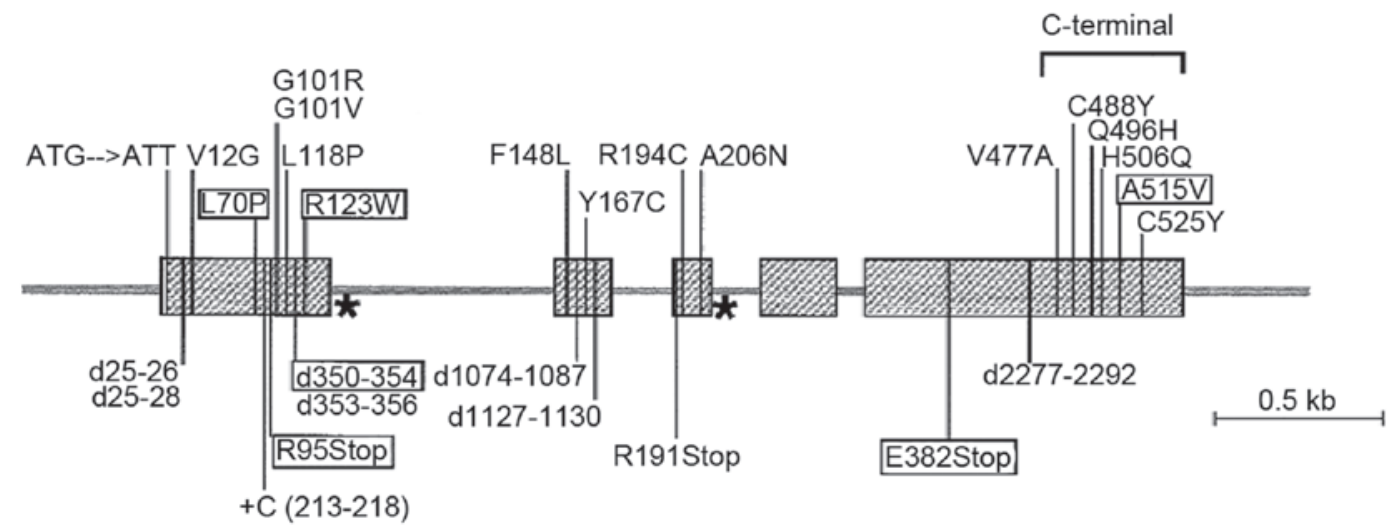

Figure 1. Anti-Müllerian hormone gene. Missense mutations are marked on the top and other mutations are marked on the bottom. Splice site mutations are marked with $\left(^{*}\right)$ and those in black boxes are common mutations.

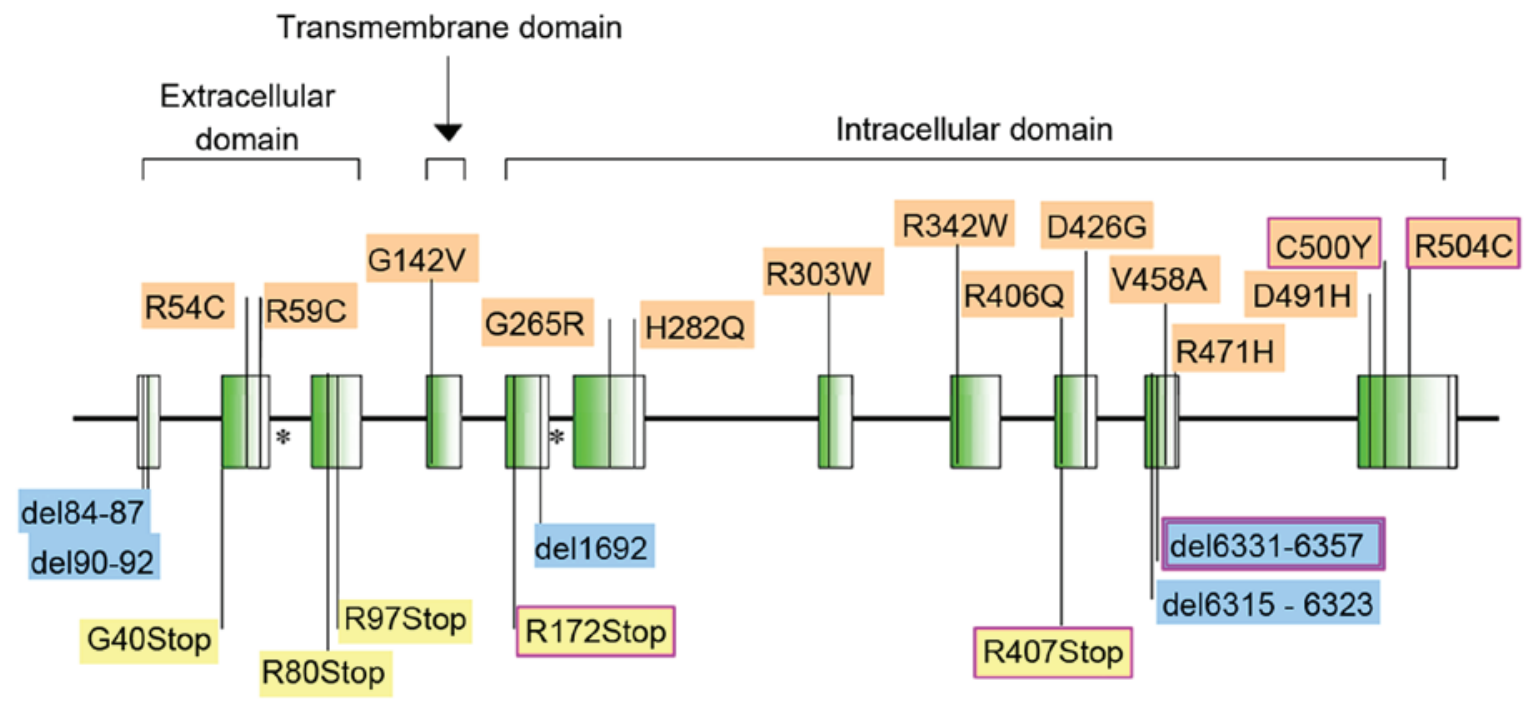

$0.5 \mathrm{kbp}$

Figure 2. Anti-Müllerian hormone type II receptor gene. Missense mutations are marked on the top and other mutations are marked on the bottom. Splice site mutations are marked with (") and those in purple boxes are common mutations.

testis descent $(20,21)$. Following the peak, AMH levels decrease as FSH levels also decrease to baseline levels. The secretion of testosterone increases following activation of the hypothalamic-pituitary-gonadal axis and in response, the androgen receptor is expressed in Sertoli cells (22). Androgens bound to androgen receptors then suppress transcription of SF-1, which leads to a decrease in AMHF expression (19,23). Tumor necrosis factor- $\alpha$ produced by testicular meiosis of spermatogenic cells activates nuclear factor (NF)- $\mathrm{kB}$ in Sertoli cells. The activated $\mathrm{NF}-\mathrm{kB}$ then enters the nucleus and binds to SF-1, the promoter of AMH, to inhibit AMH expression (24).

The development of a normal male requires testosterone, which is secreted by Leydig cells, to facilitate the development of the epididymis, sperm duct and seminal vesicles (17). A deficiency in AMH or a defect in AMHR-II in male embryos leads to the development of PMDS. The secretion and function of testosterone are not influenced during PMDS and therefore, the development of the derivatives of the Wolffian ducts and external genital differentiation occurs normally. Therefore, patients with PMDS exhibit a 46,XY chromosome pattern and MDs are present, although they exhibit a normal male phenotype. The cryptorchidism phenomenon of PMDS may be explained by decreased levels of insulin-like 3 (INSL-3), or because the Müllerian duct construct mechanically pulls the testes, thereby preventing testicular descent. INSL-3 levels were not detected in the current study; however, it is hypothesized that mechanical pulling by the MD construct may be the reason for cryptorchidism.

PMDS is primarily caused by mutations in the AMH and AMHR-II genes $(25,26)$. The AMH gene is located on chromosome $19 \mathrm{p} 13$ and is $2.75 \mathrm{kbp}$ long (8), with mutations occurring in $\sim 45 \%$ of patients with PMDS. The AMHR-II gene is located on chromosome 12q13 and is $8.7 \mathrm{kbp}$ long (8) with mutations occurring in $\sim 40 \%$ of patients with PMDS $(25,27)$. The cause of PMDS is unclear in $\sim 15 \%$ of patients, as AMH or AMHR-II gene mutations cannot be detected. PMDS may be caused by a mutation in the AMH transduction pathway in these patients $(18,25)$.

The majority of mutations in the AMH gene have been identified in patients from the Mediterranean area and Saudi 
Table I. Clinicopathological characteristics of the patient.

\begin{tabular}{lcc}
\hline Clinicopathological characteristics & Results & Normal ranges \\
\hline Age $($ months $)$ & 17 & - \\
Height $(\mathrm{cm})$ & 79 & - \\
Testicular volume $(\mathrm{ml})$ & $2 / 2$ & - \\
AST $(\mathrm{U} / \mathrm{l})$ & 32.4 & $5.0-40.0$ \\
ALT $(\mathrm{U} / \mathrm{l})$ & 14.8 & $5.0-40.0$ \\
Cortisol $(\mu \mathrm{g} / \mathrm{ml})$ & 5.84 & $5.0-25.0$ \\
ACTH $(\mathrm{pg} / \mathrm{ml})$ & 13.5 & $0-46$ \\
LH $(\mathrm{IU} / \mathrm{l})$ & 0.12 & $\leq 4.1$ \\
FSH $(\mathrm{IU} / \mathrm{l})$ & 0.74 & $\leq 5.5$ \\
T $(\mathrm{ng} / \mathrm{dl})$ & $<20.0$ & $0-21.2$ \\
E2 $(\mathrm{pg} / \mathrm{ml})$ & 28.4 & $0-20.0$ \\
PRL $(\mathrm{ng} / \mathrm{ml})$ & 4.23 & $0.6-29.0$ \\
PGN $(\mathrm{ng} / \mathrm{ml})$ & $<0.2$ & $\leq 1.3$ \\
hCG $(\mathrm{IU} / \mathrm{l})$ & $<1.0$ & $<2.5$ \\
AMH $(\mathrm{ng} / \mathrm{ml})$ & $>23$ & $1.43-11.60$ \\
INH-B $(\mathrm{pg} / \mathrm{ml})$ & 54.8 & Normal sperm production, $75-350$ \\
& & Suspicious spermatogenesis disturbance, $50-80$ \\
& & Spermatogenesis disturbance, $<50$
\end{tabular}

AST, aspartate transaminase; ALT, alanine transaminase; ACTH, adrenocorticotropic hormone; LH, luteinizing hormone; FSH, follicle stimulating hormone; T, testosterone; E2, estradiol; PRL, prolactin; PGN, progesterone; hCG, human chorionic gonadotropin; AMH, anti-Müllerian hormone, INH-B, inhibin B.

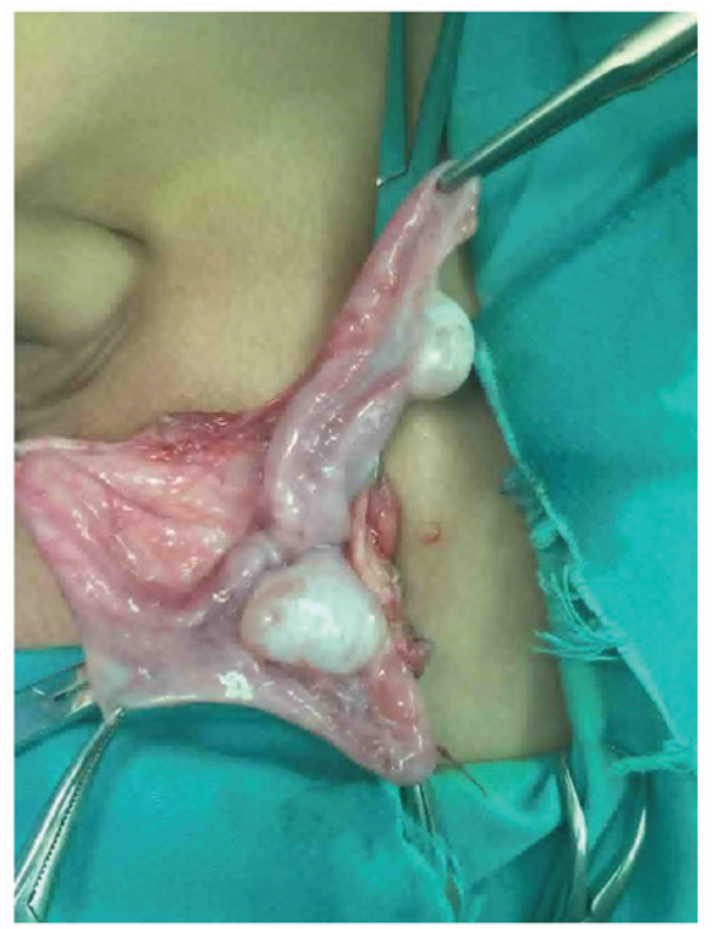

Figure 3. Uterine remnant. During surgery there was a similar kind of uterine like tissue found between the two testes.

Arabia. These are mostly missense mutations and $81 \%$ are homozygous $(8,28)$. Mutations may occur in any region of the gene apart from exon 4 , however, they most commonly arise in exon 1 (8). It has been demonstrated that the site of recurrent mutations may affect AMH processing and secretion (8). A total of 26 AMHR-II mutations have been identified to date and the majority has been identified in patients from Northern Europe and the US, with $45 \%$ of mutations being homozygous (8). The most common mutation of AMHR-II that accounts for $47 \%$ of AMHR-II mutations is del6331-6357, which is located on exon 10 and results in the deletion of $27 \mathrm{bp}(8,28)$. This mutation affects the kinase of AMHR-II, as a deletion may cause the early occurrence of a stop codon, which leads to receptor truncation following the transmembrane domain (8). To the best of our knowledge, no genetic analyses have been performed in Chinese patients.

In the current study, a novel compound heterozygous variation was identified in the patient using genetic analysis. In the mutant gene, there was a deletion of CT from c.1184 (E9) to c.1185 (E9) that originated in the father of the patient and led to a frame shift, resulting in the translation of a truncated protein. The mutation site $\mathrm{c} .1388 \mathrm{G}>\mathrm{A}$ (E10) was inherited from the mother of the patient which led to a change in $\mathrm{p} .463, \mathrm{R}>\mathrm{H}$, altering the protein structure and causing a decrease in the level of its binding. These two mutations have been identified as pathogenic by the Protein Variation Effect Analyzer (provean.jcvi.org/index.php) and SIFT (sift.jcvi.org) (29). Therefore, although they have not been reported previously, their pathogenicity may be explained.

Based on clinical expression, hormone detection and testicular biopsy are essential in the diagnosis of PMDS in adults; however, there are fewer cases of PMDS diagnosed in children due to naturally high levels of AMH in prepubescent 
boys, which makes diagnosis more challenging (17,30). There are no specific clinical symptoms, hormones or ultrasonic tests for PMDS; therefore diagnosis is challenging for pediatricians, particularly if they lack experience. It is also difficult to distinguish PMDS from partial testicular dysfunction (31). Therefore, further genetic mutation analysis is required when diagnosing PMDS in children. The patient in the current case report presented with an empty right scrotum and left inguinal mass, which differs from the symptoms identified in adult with PMDS, which include sterility, oligospermia and inguinal hernia. The results of the physical examination indicated an empty right scrotum, the top of the left scrotum reached the testis, a normal sized external genital organ and no hypospadias. In addition, AMH levels were increased whereas levels of other hormones tested were within normal ranges for a pre-pubescent boy. It should be noted that testosterone levels in adults diagnosed with PMDS are usually within normal ranges. During ultrasonic examination of the testis and pelvis, a left transverse testicular ectopia and uterine hyperechoic line were identified behind the left side of the bladder and the MD structure inside the pelvic cavity was examined, supporting the diagnosis of PMDS. A karyotyping test was subsequently performed to determine the chromosomal sex and the results determined that the patient's chromosome karyotype was $46, \mathrm{XY}$. Based on the symptoms of the patient, it was suspected that the patient had PMDS. Genetic analysis was then conducted with permission from the patient's parents and a mutation in the AMHR-II gene was identified, confirming the diagnosis of PMDS. Left transverse testicular ectopia orchidopexy also confirmed diagnosis and during surgery, there was a similar type of uterine like tissue identified between the two testes.

Remnants of MD and ectopic testis must be managed for effective treatment of PMDS. The primary aim of treatment is to maintain the patient's fertility, as well as prevent or detect the malignant transformation of the MDs and ectopic testis early on. It has been demonstrated that abnormalities in the number of germ cells of convoluted seminiferous tubules in patients with cryptorchidism begins at the age of 1 year and the damage becomes permanent after the age of 2 . Undescended testes in the inguinal canal and abdomen hinder the production of sperm; therefore an orchidopexy is required by the age of 2 (32). In cases of cryptorchidism, the testes typically descend spontaneously for those $<2$ years old, therefore it is recommended that orchidopexy only be conducted in patients aged $>2$ years.

It has been demonstrated that there is an improvement in the fertility of patients with intra-abdominal testis that undergo surgery prior to puberty. If the cord is long enough, an orchidopexy may be performed so that the spermatic cord brings the testicle down to the scrotum and the testicle can be fixed there. Follow-up should then be performed following surgery. If the cord is too short or separation of one of the arteries is unsuccessful in creating a longer cord, the testicle may be placed under the skin in the groin region and gonadotropin treatment may be used so to promote testicular descent. If testicles remain undescended, orchiectomy should not be considered. If the testicle was located in the groin, it is easier to be observed in case malignant transformation and other complications. Fertility is maintained in only $14 \%$ of patients with intra-abdominal testis who are diagnosed and treated following puberty (32). The malignant transformation rate is also increased compared with those treated prior to puberty, therefore, an orchiectomy may be considered. However, this is a serious procedure and it is necessary to discuss this with children and their parents due to the requirements of lifelong androgen treatment following this procedure in childhood $(31,33)$. This procedure is also controversial due to the low malignancy rate for ectopic testis in the inguinal hernia sac; therefore an orchidopexy may be performed $(9,34)$. A lifetime of androgen replacement therapy is required when testicle atrophy occurs, the testicles become cancerous or following removal of the testes (33). If malignant transformation has already occurred, removal of the testes must be performed, in addition to radiation therapy and chemotherapy (25). The overall incidence of malignant transformation is $15 \%(17,30)$. The higher the position of the testes, the higher the risk of malignant transformation in patients with PMDS with cryptorchidism, however, there is no difference in the malignant transformation rate between the two sides of the ectopic testes (32). The results of previous reviews demonstrate that malignant transformation occurs 6-18 years following cryptorchidopexy. However, there is no correlation between onset of malignant transformation and surgical treatment or patient age $(35,36)$. This indicates that the risk of malignant transformation is congenital and does not depend on external causes.

Treatment of the remnants of MD remains controversial. Previous studies have demonstrated that children with PMDS require removal of $\mathrm{MD}$ remnants due to malignant tumors identified in Müllerian remnants and the MD being connected with the seminal vesicle causing urinary tract infections, periodic hematuria, stones and urination disorders $(25,27)$. However, other studies have suggested retaining Müllerian remnants to prevent damage to the vas deferens and disruption of collateral blood supply to the testes $(25,27)$.

A long-term management strategy includes assessment of testicular function and monitoring of malignant transformation at an earlier stage $(35,36)$. A total of 30 cases of malignant transformation have been identified in patients with PMDS (2). The majority of malignant tumors are identified in ectopic testes and few originate from the Müllerian remnants. Only one patient has been reported to develop adenocarcinoma of endocervical origin (37) and one other patient developed clear cell adenocarcinoma (38). Testicular tumors include seminomas, embryonic cell carcinoma, yolk sac tumors and teratomas, of which seminomas are the most common (2). In China, there has only been one case of malignant seminoma reported (39). The long-term growth, development and fertility of patients with PMDS must be considered prior to removal of non-functional MD structures.

In conclusion, PMDS is a rare disease. Due to the lack of distinct clinical features at an early stage, PMDS is usually diagnosed when children are tested for other diseases, including cryptorchidism and inguinal hernia. The early diagnosis and treatment of PMDS in children is therefore challenging. Correct diagnosis depends on a systematic endocrine assessment and genetic analysis. The case presented in the current study furthers knowledge of the gene profile of PMDS. However, removal of MD structures from patients with PMDS as a treatment strategy remains controversial and further studies investigating its impact are required. 


\section{Acknowledgements}

The present study was funded by The National Key Research and Development Program of China (grant no. 2016YFC0901505) and Beijing Municipal Science and Technology Funding (grant no. Z151100003915103).

\section{References}

1. Smith-Harrison LI, Patel MS, Smith RP and Schenkman NS: Persistent Mullerian duct structures presenting as hematuria in an adult: Case report of robotic surgical removal and review of the literature. Urol Ann 7: 544-546, 2015.

2. Barad AK, Bharath NM, Narayana V, Raja VO and Jambula PR: Persistent Mullerian duct syndrome with Embryonal cell carcinoma along with Ectopic cross fused kidney. J Clin Diagn Res 10: PD07-PD08, 2016.

3. Clarnette TD, Sugita Y and Hutson JM: Genital anomalies in human and animal models reveal the mechanisms and hormones governing testicular descent. Br J Urol 79: 99-112, 1997.

4. Shalaby MM, Kurkar A, Zarzour MA, Faddan AA, Khalil M and Abdelhafez MF: The management of the persistent Mullerian duct syndrome. Arab J Urol 12: 239-244, 2014.

5. Nerune SM, Hippargi SB, Mestri NB and Mehrotra NM: Persistent Mullerian duct syndrome with ovarian endometriosis-a rare case report. J Clin Diagn Res 10: ED14-ED15, 2016.

6. Shen J and Bi YL: Laparoscopic management of Persistent Müllerian duct combined with cystoscope. J Clin Pediat Surg 12: 107-109, 2013

7. Ju XB, Qian LX, Zhang W, Wu HF, Xu ZQ and Sui YG: Persistent Müllerian duct syndrome: report of two cases. Chin J Surg 45: 863-864, 2007

8. di Clemente $\mathrm{N}$ and Belville C: Anti-Mullerian hormone receptor defect. Best Pract Res Clin Endocrinol Metab 20: 599-610, 2006

9. Fonkalsrud EW: Current management of the undescended testis. Semin Pediatr Surg 5: 2-7, 1996.

10. Marshall WA and Tanner JM: Variations in the pattern of pubertal changes in boys. Arch Dis Child 45: 13-23, 1970.

11. Nilson O: Hernia Uteri Inguinalis beim manne. Aeta Chir Scand 83: 231, 1939.

12. Palanisamy S, Patel ND, Sabnis SC, Palanisamy N, Vijay A and Chinnusamy P: Laparoscopic hysterectomy with bilateral orchidectomy for Persistent Mullerian duct syndrome with seminoma testes: Case report. J Minim Access Surg 11: 273-275, 2015.

13. Mohapatra M and Subramanya YS: Persistent Müllerian duct syndrome of mixed anatomical variant (combined male and female type) with mixed germ cell tumor of left intra-abdominal testis. Indian J Pathol Microbiol 59: 212-215, 2016.

14. Mohammadi Sichani M, Heidarpour M, Dadkhah A and Rezvani M: Persistent mullerian duct syndrome with an irreducible inguinal hernia. Urol J 6: 298-300, 2009.

15. Dekker HM, de Jong IJ, Sanders J and Wolf RF: Persistent Mullerian duct syndrome. Radiographics 23: 309-313, 2003.

16. Kamble RS, Gupta RK, Gupta AR, Kothari PR, Dikshit KV and Kesan KK: Laparoscopic management of transverse testicular ectopia with persistent mullerian duct syndrome. J Minim Access Surg 11: 213-215, 2015.

17. Renu D, Rao BG, Ranganath K and Namitha: Persistent mullerian duct syndrome. Indian J Radiol Imaging 20: 72-74, 2010.

18. Belville C, Josso N and Picard JY: Persistence of Müllerian derivatives in males. Am J Med Genet 89: 218-223, 1999.

19. Matuszczak E, Hermanowicz A, Komarowska M and Debek W: Serum AMH in physiology and pathology of male gonads. Int J Endocrinol 2013: 128907, 2013.

20. Grinspon RP and Rey RA: Anti-müllerian hormone and sertoli cell function in paediatric male hypogonadism. Horm Res Paediatr 73: 81-92, 2010.
21. Wang F, Zhang H, Liu QX, et al: A clinical study on the detection of serum anti-Mullerian hormone and inhibin B in 1400 cases of healthy children aged 0 to 5 years. Chin J Practical Pediatric 31: 450-454, 2016.

22. Teixeira J, Maheswaran S and Donahoe PK: Müllerian inhibiting substance: An instructive developmental hormone with diagnostic and possible therapeutic applications. Endocr Rev 22: 657-674, 2001.

23. Rey R, Lukas-Croisier C, Lasala C and Bedecarrás P: AMH/MIS: What we know already about the gene, the protein and its regulation. Mol Cell Endocrinol 211: 21-31, 2003.

24. Aksglaede L, Sorensen K, Boas M, Mouritsen A, Hagen CP, Jensen RB, Petersen JH, Linneberg A, Andersson AM, Main KM, et al: Changes in anti-Müllerian hormone (AMH) throughout the life span: A population-based study of 1027 healthy males from birth (cord blood) to the age of 69 years. J Clin Endocrinol Metab 95: 5357-5364, 2010.

25. Patil V, Muktinaini S, Patil R and Verma A: Persistent mullerian duct syndrome: A case report. Indian J Surg 75 (Suppl 1): S460-S462, 2013.

26. Hoshiya M, Christian BP, Cromie WJ, Kim H, Zhan Y, MacLaughlin DT and Donahoe PK: Persistent Mullerian duct syndrome caused by both a 27-bp deletion and a novel splice mutation in the MIS type II receptor gene. Birth Defects Res A Clin Mol Teratol 67: 868-874, 2003.

27. Manjunath BG, Shenoy VG and Raj P: Persistent mullerian duct syndrome: How to deal with the mullerian duct remnants-a review. Indian J Surg 72: 16-19, 2010.

28. Imbeaud S, Belville C, Messika-Zeitoun L, Rey R, di Clemente N, Josso N and Picard JY: A 27 base-pair deletion of the anti-müllerian type II receptor gene is the most common cause of the persistent müllerian duct syndrome. Hum Mol Genet 5: 1269-1277, 1996.

29. Cheng J, Lin R, Zhang W, Liu G, Sheng H, Li X, Zhou Z, Mao X and Liu L: Phenotype and molecular characteristics in 45 Chinese children with $5 \alpha$-reductase type 2 deficiency from South China. Clin Endocrinol (Oxf) 83: 518-526, 2015.

30. Prakash N, Khurana A and Narula B: Persistent Müllerian duct syndrome. Indian J Pathol Microbiol 52: 546-548, 2009.

31. Wang SH, Jiang DP and Li ZZ: Research progress of persistent Müllerian duct syndrome. Chin J Pediatr Surg 37: 73-76, 2016.

32. Shao FM, Mao QL, Yang HM and Hou LJ: Persistent Mullerian duct syndrome: A report of two cases and literature review. Chin J Pediateic Surg 32: 468-470, 2011.

33. Odi TO, Abdur-Rahman LO and Nasir AA: Persistent Mullerian duct syndrome: A case report and review of the literature. Afr J Paediatr Surg 7: 191-193, 2010.

34. Batata MA, Whitmore WF Jr, Chu FC, Hilaris BS, Loh J, Grabstald H and Golbey R: Cryptorchidism and testicular cancer. J Urol 124: 382-387, 1980.

35. Manassero F, Cuttano MG, Morelli G, Salinitri G, Spurio M and Selli C: Mixed germ cell tumor after bilateral orchidopexy in persistent Mullerian duct syndrome with transverse testicular ectopia. Urol Int 73: 81-83, 2004.

36. Melman A, Leiter E, Perez JM, Driscoll D and Palmer C: The influence of neonatal orchiopexy upon the testis in persistent Mullerian duct syndrome. J Urol 125: 856-858, 1981.

37. Thiel DD and Erhard MJ: Uterine adenosarcoma in a boy with persistent mullerian duct syndrome: First reported case. J Pediatr Surg 40: e29-e31, 2005.

38. Shinmura Y, Yokoi T and Tsutsui Y: A case of clear cell adenocarcinoma of the müllerian duct in persistent mullerian duct syndrome: The first reported case. Am J Surg Pathol 26: 1231-1234, 2002

39. Yu DX, Fang WH and Jiang S: Persistent Müllerian duct syndrome with transverse testicular ectopia (report of 2 cases). Chin J Urol 23: 678-680, 2002. 